\title{
Optimal Preparation for Safe Airway Management During Covid-19
}

\author{
Hassan Shaaib ${ }^{1 *}$ and Muhammad Ali Abbas ${ }^{2}$ \\ ${ }^{1}$ Lecturer of Anesthesia Technology in Prince Sultan Military College of Health \\ Sciences, Dhahran, Saudi Arabia \\ ${ }^{2}$ Consultant Anaesthetist in Prince sultan military college of health sciences Dharan, \\ Saudi Arabia \\ *Corresponding Author: Hassan Shaaib, Lecturer of Anesthesia Technology in \\ Prince Sultan Military College of Health Sciences, Dhahran, Saudi Arabia.
}

DOI: 10.31080/ASMS.2020.04.0756
Received: August 17, 2020

Published: September 30, 2020

(C) All rights are reserved by Hassan Shaaib and Muhammad Ali Abbas.

\section{Abstract}

Airway management is the core skill of anesthesiologists, intensive care units, and emergency care providers. Clinicians assured that oxygenation is not possible without an open and safe airway. The Severe Acute Respiratory Syndrome-Corona-2 Virus (SARSCoV-2), which causes COVID-19, is encapsulated with single-stranded ribonucleic acid and is highly contagious. Authors believed that the transfer of the virus occurs primarily through direct contact with the patient or contaminated surfaces or through the spread of droplets (i.e., relatively large particles deposited in the air) and smaller particles staying longer in the air. Any airway management or procedures in emergency, operation theatre, and intensive care departments can produce aerosols to increase the risk of contamination. For the above reason, healthcare professionals who are treating patients with COVID-19 are at risk of developing the disease. The purpose of the current study is to review the methods that reduce the risk of an airborne viral spread, maximize the early attempt success, and encourage the use of indirect glottic visualization with video laryngoscopy (VL). The mentioned measures are approved to reduce personnel exposure in all adult COVID 19 patients who are requiring airway management in all emergency departments, hospital wards, and ICUs. The author highlights the needed measures that require to be taken for the safety of the health care workers associated with the airway procedure while providing high-quality patient care to the COVID-19, suspected, or confirmed patients.

Keywords: Airway; COVID-19; Management; Optimal; Safe; Preparation; Health Care Workers

\section{Introduction}

Airway management is the core skill of anesthesiologists, intensive care units, and emergency care providers. And oxygenation is not possible without an open and safe airway [1]. In December 2019, the outbreak of a novel coronavirus (SARS-CoV-2) has caused an international acute respiratory illness termed COVID-19 $[2]$.

A confirmed or suspected (COVID-19) patient, who needs tracheal intubation is a unique challenge, as its activity is a potentially infectious aerosol-generating approach, and this may subject the team of intubation to risks [3]. All anesthesia and other profes- sionals have to consider all measures that limit the likelihood of aerosolization of droplet particles. As stated by the guidelines recommended by the American Society of the anesthesiologist (ASA), these guidelines include the selection of the most experienced anesthesia provider available to fulfill the intubation, as manageable. Besides wearing personal protective equipment (PPE), which provides safety, like suitable tested N95 mask, or a powered airpurifying respirator (PAPR), face shield or goggles as well as gown and gloves [4]. The avoidance of awake fiberoptic intubation would be a must unless emergency indicated. The consideration of a rapid sequence induction (RSI) is advised to avoid manual ventilation of the patient's lungs and potential aerosolization. Small tidal volume is applied if required [5]. 
Airway healthcare providers should now already be involved in regular intensive training for the airway management of the COVID- 19 patient groups. The focus of the training should rely on the principles of early intervention, careful planning, cautious infection control, efficient operation, comprehensive communication, and standardized practice [6].

This review aims to provide healthcare workers with a well-organized approach to manage the airway for the COVID 19 patients in emergency and Intensive care departments, to protect them and optimize and increase the chances of successful first attempt intubation.

Staff protection during airway management of COVID-19 patient

It is recommended that all medical personnel who are involved in the care of patients suspected of COVID-19 must comply with airborne precautions, hand hygiene, and the wearing of personal protective equipment. All aerosol-generating procedures should be performed in an airborne infection isolation room. Double gloving as standard can provide additional protection and minimize spread to surrounding material by fomite contamination after intubation [7]. The highest viral load of SARS - CoV - 2 occurs in sputum and upper respiratory tract secretions. Tracheal intubation is a high-risk procedure for the airway manager, especially when there is a high risk of viral load exposure and contamination to healthcare workers. It is associated with more severe diseases. Therefore, airway managers should take appropriate measures. This area is critical, and personal protective equipment is part of a system to reduce viral exposure. There are many regularly updated recommendations on preventing and controlling COVID-19 infections [8-10]. Recent publications have issued several similar reports describing the use of various "airway boxes" or drapes aimed at providing a physical barrier to aerosols and droplets [11]. Researchers said that most of the viral spread is due to aerosols or airborne particles produced by asymptomatic carriers in the air during speech and breathing. Airborne spread from people unaware that they have been infected highlights the importance of mass testing, masking, and physical distance to reduce the spread of the virus. Masks are effective in reducing airborne contamination of SARS-CoV-2. An adequately fitted cover provides a healthy physical barrier to reduce the number of microbes in the breath. Surgical and N95 masks are inadequate, and priority should be given to front lines like healthcare professionals [12]. The increased risk of infection by healthcare professionals with multiple airway manipulation requires the use of reliable airway techniques that maximize first-pass success and be applicable to saving techniques. No argument for alone operators, but personnel who are not directly involved in the airway procedure should not be unnecessarily in the room where airway management is taking place. You will probably need three people: an intubator, an assistant, and a third person to give medication and check the monitors. A runner should stay outside and be able to call for help if needed quickly (Figure 4) [8].

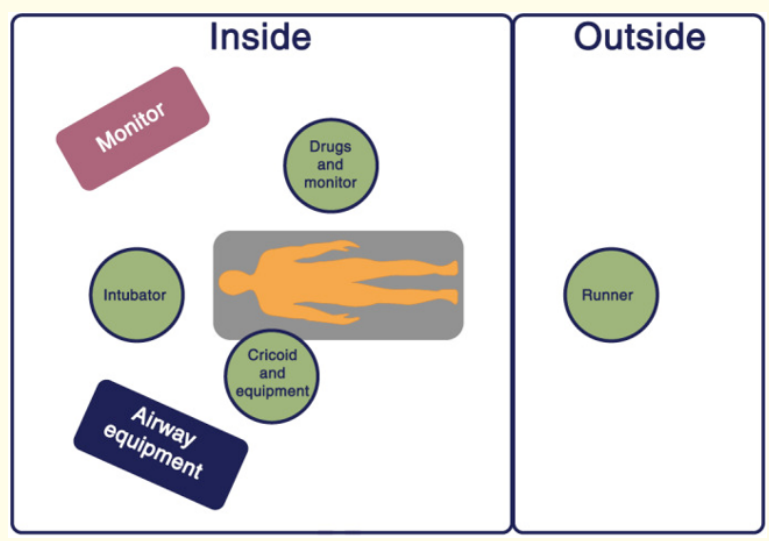

Figure 1: Personnel plan for tracheal intubation of a patient with coronavirus disease 2019. Adapted from [13].

\section{Choices of airway device during COVID-19}

The goal of airway management is achieved by endotracheal intubation, which is quickly open the airways on the first try and reduce or eliminate the aerosolization of respiratory secretions. The primary considerations when intubating are: to minimize the number of people in the room during intubation, ensure optimal patient placement for intubation, and have the most experienced clinician perform the intubation only after have worn double gloves during intubation. The use of video laryngoscopy is preferred for rapid procedures. If you need artificial ventilation, another option is the use of supraglottic airways. Also, mask ventilation, if required, in which the use of two-handed technique and tidal volumes at low pressure is recommended. Authors preferred the Use of end-tidal carbon dioxide $\left(\mathrm{ETCO}_{2}\right)$ and an appropriate tube depth during video laryngoscopy to verify endotracheal tube placement rather than bilateral breath sounds. Besides, dispose of contaminated equipment immediately. For ventilated patients who do not breathe spontaneously, leave the filter in the ETT [13]. 
The aerosol box for intubation in coronavirus disease 2019 patients

In the light of PPE deficiencies, solutions such as increased PPE production, and recommendations for reuse of PPE, and in some cases, the creation of new devices to protect health workers. While such innovations are particularly useful in the face of supply shortages, the risks inherent in this approach are also explained. One such new device is the aerosol box. Lai Hsien-Yung first announced the device, which consists of a transparent plastic cube that covers the patient's head and shoulders. The intubation specialist has an access hole for the arm, and there may be an additional hole for the assistant figure [2]. Some studies present significant patient safety concerns in the use of aerosol boxes for the intubation of COVID-19 patients. However, aerosol boxes may paradoxically increase the risk for physicians participating in COVID-19 airway management by causing a PPE violation $[14,15]$.
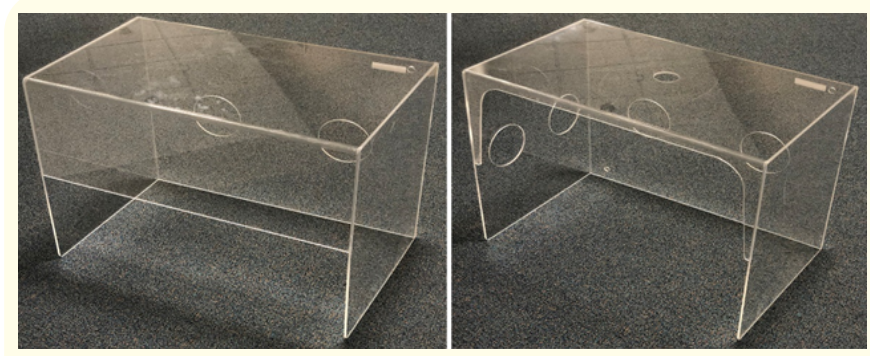

Figure 2: Source $[15,16]$.

"The early-generation aerosol box (left) and the latest-generation aerosol box (right) were studied. Dimensions of both boxes were the same: $65-\mathrm{cm}$ wide, $50-\mathrm{cm}$ tall, and $40-\mathrm{cm}$ deep. The primary armholes are $12.5 \mathrm{~cm}$ in diameter and positioned identically in both boxes".

\section{Methods of laryngoscopy during COVID-19}

Laryngoscopes are devices used to perform direct laryngoscopy and to aid in tracheal intubation [16]. Tracheal intubation, supported by video laryngoscopy, has become an alternative to traditional laryngoscopes recently (Figure 3) [16,17]. The authors suggested the use of video laryngoscopy as the method of choice for airway management in patients during the COVID-19 outbreak, to increase the distance from the operator to the patient's mouth. A technique that similarly increases the distance between the pa-

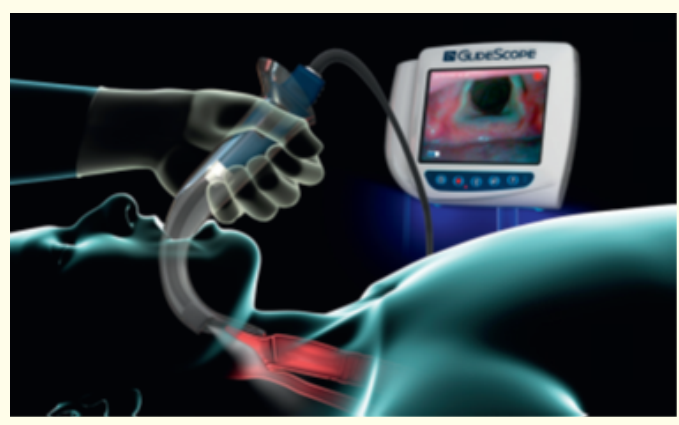

Figure 3: The Glideslope video laryngoscope. (Courtesy of Verathon Medical UK Ltd.) $[17,18]$.

tient and operator is flexible bronchoscopic intubation (by experienced operators) under anesthesia with deep muscle relaxation. The authors also emphasized that if a video laryngoscope is used, it is preferential not to use its integrated oxygen channel if present as the extraneous flow of oxygen may increase aerosol generation rather than reduce it. Nor should stylets or flexible bronchoscopes have oxygen insufflated through their central channels [18].

Confirming the correct endotracheal tube placement for COVID-19 patient

It is dangerous to rely on seeing the endotracheal tube passing through the vocal cords as clinical signs. Capnography should be considered mandatory for any patient requiring intubation, especially in an emergency. The endotracheal tube should be replaced immediately for each patient, not in cardiac arrest without $\mathrm{ETCO}_{2}$ detected [19]. Many methods are available to confirm endotracheal tube (ETT) placement and have varying degrees of efficacy and completion times. Ultrasound (US) tracheal intubation can be very accurate in determining the location of ETT in real-time in intensive care patients undergoing emergency intubation [20]. Stethoscopes are known to have the potential to spread virulent pathogens and appear to play a more significant role in the intensive care unit. The current outbreak of COVID19 disease poses severe risks for the healthcare provider involved in initiating invasive ventilation for ARDS patients. The medical staff is infected every day, and many colleagues die from the new disease. Since surface contamination is one of the common ways of spreading the disease, the stethoscope, which is a prevalence tool that should be in contact with infected patients, may play a role in the cephalic limb of the healthcare professional important. Auscultation of both lungs with a stethoscope 
remains the standard care in protocols worldwide. The concern of including ultrasound as a tool for airway management is not new, and it has been shown that ultrasound has better sensitivity and specificity than auscultation [21]. In recent years, the use of ultrasound (US) to confirm endotracheal tube (ETT) placement has gained popularity. Various techniques are already available to confirm the placement of the endotracheal tube. However, each has its limits, and some are not always available in the emergency room. The likely reason why the US airways attract attention is the ease of getting pictures. For ETT approval, Airway US is best used when the tidal CO2 monitor, radiology is not available, the patient is intubated and requires airway approval or does not respond as expected after intubation [22]. Among the advantages of the Tracheal intubation confirmed by ultrasound, it is highly sensitive and specific in obese patients [23].

\section{Extubation during COVID-19}

Experts developed a technique to minimize exposure of staff in proximity during extubation-the 'mask over tube' technique, which is described by using a second airway filter. The use of the second airway filter is critical to avoid anesthetist from exposing to direct droplets or aerosols produced by extubation or associated coughing (Figure 4) [24].

Barrier limits have been proposed, with a mixed response in the literature to reduce the risk of personnel exposure during high-risk procedures; the first known hood was described as a "spray pox" built-in resistant plexiglass. This device was intended for use in low resource situations where PPE was not sufficiently available for healthcare professionals involved in high-risk procedures. Canelli., et al. evaluated a fume hood of this type. Which established in a simulated intubation experiment and assured that this fume hood prevented the spread of aerosol particles [25,26]. Finally, after confirming that the anesthetic face mask is no longer needed, after extubation, the patient is recommended to wear a surgical mask. Supplemental oxygen can be given under the surgical mask with nasal prongs. Staff must verify that the integrity of the PPE is preserved. Removal of gloves can only be done if the patient has been transferred to another staff member. Depending on the ventilation of the room, the room requires further action for a variable time after an aerosol treatment [24].

\section{Conclusion}

Managing known or suspected COVID-19 patients need special consideration for staff and patient safety. Accuracy is paramount,
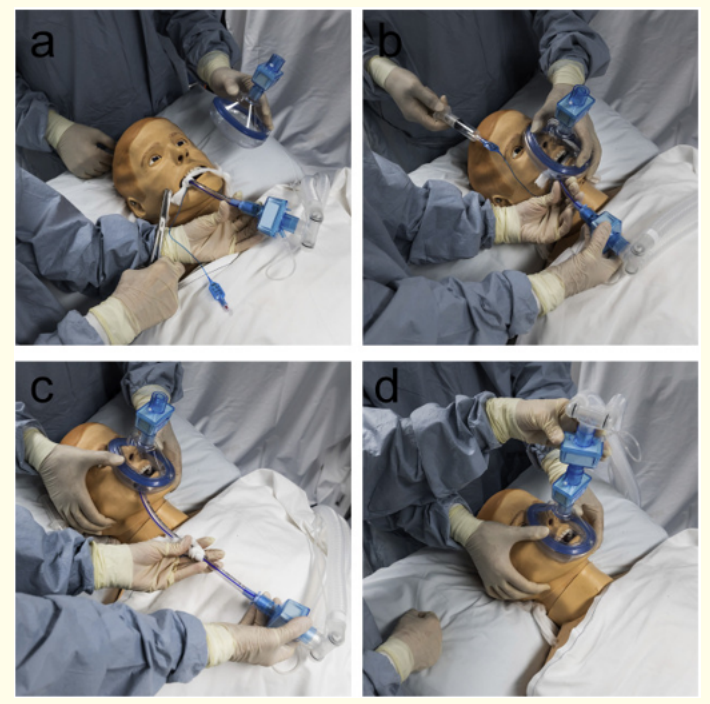

Figure 4: Mask-over tube extubation technique [25]. "(a) Tracheal tube positioned at one corner of the mouth with ties cut. (b) Facemask with airway filter positioned to create a seal over the face and tracheal tube. (c) The assistant withdraws the tracheal tube from under the side of the facemask using a two-handed technique to control the tracheal tube. (d) The tracheal tube has been detached, and the circuit has been connected to the second airway filter on the facemask" [25].

and clinicians need to avoid unreliable, unknown, or repetitive techniques during airway management to ensure they are safe, accurate, and fast. Immediate maintenance means not being in a hurry and being on time, without delay as well. The review emphasized the principles that can achieve these goals; the details of these principles may change as new evidence emerges.

\section{Conflict of Interest}

No conflict of interest.

\section{Bibliography}

1. Matioc AA. An Anesthesiologist's Perspective on the History of Basic Airway Management: The "modern" Era, 1960 to Present. Vol. 130, Anesthesiology. Lippincott Williams and Wilkins (2019): 686-711. 
2. Zhang X., et al. "Safe and effective management of tracheostomy in COVID-19 patients". In: Head and Neck. John Wiley and Sons Inc. (2020).

3. Ahmad I., et al. "Awake tracheal intubation in a suspected COVID -19 patient with critical airway obstruction". Anesthesia Reports 8.1 (2020): 28-31.

4. Liverman CT., et al. "The Use and Effectiveness of Powered Air Purifying Respirators in Health Care: Workshop Summary" (2015).

5. COVID-19: American Society of Anesthesiologists Recommendations for Anesthesia Management - Ambulatory Surgery Center Association (ASCA).

6. Brewster DJ., et al. "Consensus statement: Safe Airway Society principles of airway management and tracheal intubation specific to the COVID-19 adult patient group". Medical Journal of Australia 212.10 (2020): 472-481.

7. Cheung JCH., et al. "Staff safety during emergency airway management for COVID-19 in Hong Kong". The Lancet Respiratory Medicine. Lancet Publishing Group 8 (2020): e19.

8. Cook TM., et al. "Consensus guidelines for managing the airway in patients with COVID-19: Guidelines from the Difficult Airway Society, the Association of Anaesthetists the Intensive Care Society, the Faculty of Intensive Care Medicine and the Royal College of Anaesthetist". Anaesthesia 75.6 (2020): 785799.

9. Wang W., et al. "Detection of SARS-CoV-2 in Different Types of Clinical Specimens". JAMA - Journal of the American Medical Association 323 (2020): 1843-1844.

10. Wu Z and McGoogan JM. "Characteristics of and Important Lessons from the Coronavirus Disease 2019 (COVID-19) Outbreak in China: Summary of a Report of 72314 Cases from the Chinese Center for Disease Control and Prevention". JAMA - Journal of the American Medical Association 323 (2020): 1239-1242.

11. Gould CL., et al. "Protecting staff and patients during airway management in the COVID-19 pandemic: are intubation boxes safe?" British Journal of Anaesthesia (2020).
12. Gould CL., et al. "Protecting staff and patients during airway management in the COVID-19 pandemic: are intubation boxes safe?". British Journal of Anaesthesia. Elsevier Ltd (2020).

13. Coronavirus disease 2019 (COVID-19): Anesthetic concerns, including airway management and infection control - UpToDate.

14. News KE-T, 2020 undefined. Taiwanese doctor invents device to protect US doctors against coronavirus.

15. Begley JL., et al. "The aerosol box for intubation in coronavirus disease 2019 patients: an in-situ simulation crossover study". Anaesthesia 75.8 (2020): 1014-1021.

16. Smith G and Conway CM. "Anaesthetic equipment". British Journal of Anaesthesia 57 (1985): 639.

17. Liao CC., et al. "Video laryngoscopy-assisted tracheal intubation in airway management". Expert Review of Medical Devices. Taylor and Francis Ltd 15 (2018): 265-275.

18. Singh N., et al. "Video laryngoscopy in COVID-19 patients: a word of caution". Canadian Journal of Anesthesia Springer (2020): 1-1.

19. Black JJM and Skinner D V. "Confirmation of correct endotracheal tube placement (multiple letters)". British Association for Accident and Emergency Medicine 17 (2000): 74-75.

20. Arya R., et al. "Real-time Tracheal Ultrasound for the Confirmation of Endotracheal Intubations in the Intensive Care Unit: An Observational Study". Journal of Ultrasound in Medicine 38.2 (2019): 491-497.

21. Dîrzu DS. "Replacing the stethoscope with ultrasound identification of lung sliding to confirm correct endotracheal tube placement for COVID19 patients - time for a paradigm shift?" International Journal of Clinical Practice (2020).

22. ACEP // American College of Emergency Physicians.

23. Men XQ and Yan XX. "Tracheal Ultrasound for the Accurate Confirmation of the Endotracheal Tube Position in Obese Patients". Journal of Ultrasound in Medicine 39.3 (2020): 509-513. 
24. D'Silva DF, et al. "Extubation of patients with COVID-19". British Journal of Anaesthesia. Elsevier Ltd 125 (2020): e192-195.

25. Kangas-Dick AW., et al. "Safe extubation during the COVID-19 pandemic". Respiratory Medicine. W.B. Saunders Ltd 170 (2020): 106038.

26. Weaning and Extubation Guideline.

\section{Assets from publication with us}

- Prompt Acknowledgement after receiving the article

- Thorough Double blinded peer review

- Rapid Publication

- Issue of Publication Certificate

- High visibility of your Published work

Website: www.actascientific.com/

Submit Article: www.actascientific.com/submission.php

Email us: editor@actascientific.com

Contact us: +919182824667 